\title{
Radiolabeled neurogenesis marker imaging: A revolution in the neurological diseases management?
}

\author{
Reza Nemati ${ }^{a}$, Somayeh Mehdizadeh ${ }^{a}$, Iraj Nabipour ${ }^{\mathrm{b}}$, Hooman Salimipour ${ }^{\mathrm{a}}$, Darioush Iranpour ${ }^{\mathrm{b}}$, \\ Majid Assadi ${ }^{\text {, } *}$ \\ a Department of Neurology, Faculty of Medicine, Bushehr University of Medical Sciences, Bushehr, Iran \\ ${ }^{\mathrm{b}}$ Department of Biochemistry, The Persian Gulf Marine Biotechnology Research Center, Bushehr University of Medical Sciences, Bushehr, Iran \\ ${ }^{\mathrm{c}}$ The Persian Gulf Nuclear Medicine Research Center, Bushehr University of Medical Sciences, Bushehr, Iran
}

\section{A R T I C L E I N F O}

\section{Article history:}

Received 8 August 2013

Accepted 30 November 2013

\begin{abstract}
A B S T R A C T
A reduced rate of neurogenesis occurs in the adult brain of patients with neurological diseases, with the rate of new neuron proliferation not sufficient to replace neuron loss. Neurogenesis can be induced by several factors, including basic fibroblast growth factor, epidermal growth factor, and brain-derived neurotrophic factor.

Neurogenesis determination is a valuable parameter for determining disease progression and monitoring various treatments. Currently, neurogenesis detection is possible by invasive methods, such as bromodeoxyuridine (BrdU) cell labeling and immunohistological analysis of immature neuron markers. However, these are not compatible with alive model examination. Neurogenesis detection by noninvasive methods, such as radiolabeling of specific antibodies and scintigraphy imaging, could shed light on immature neuronal markers.

We propose that brain scintigraphy after radiolabeling of a specific antibody of an immature neuronal marker is a useful new modality for neurogenesis detection and that it would aid the management of neurological diseases.
\end{abstract}

(c) 2013 Elsevier Ltd. All rights reserved.

\section{Introduction}

Neuron loss is deemed to be irreversible in the adult human brain because brain tissue, unlike other tissue, has a limited inability to replace neurons with new cells. Progressive neuron loss eventually results in neurological diseases, such as Parkinson's disease and Alzheimer's disease [1]. Although neural stem/progenitor cells (NPCs), defined as primitive cells, are abundant in different discrete regions of the brain during embryonic and postnatal development, the adult brain contains these NPCs in a limited number of brain regions, including the subgranular zone (SGZ) in the dentate gyrus and the subventricular zone (SVZ) in the lateral walls of the lateral ventricles where NPCs migrate and form neurons in the hippocampal dentate gyrus and the olfactory bulb, respectively [1].

Proliferation and neurogenesis occur in all vertebrates, including mammals, following central nervous system (CNS) tissue damage to replace and repair the injured nerves [2]. Several studies have reported that neurogenesis can be induced by stress, the envi-

\footnotetext{
* Corresponding author. Address: Department of Nuclear Medicine, The Persian Gulf Biomedical Sciences Institute, Boostan 19 Alley, Sangi Street, Bushehr, Iran. Tel.: +98 771 2580169; fax: +98 7712541828 .

E-mail addresses: assadipoya@yahoo.com, asadi@bpums.ac.ir (M. Assadi).
}

ronment, and exercise, as well as by various CNS diseases, such as seizure and stroke [3,4].

Several methods have been used to detect neurogenesis, such as bromodeoxyuridine (BrdU) cell labeling, incorporation of thymidine analogs, retroviral labeling, and immunohistological analysis of immature neuron markers [3,4]. However, these techniques are invasive and cannot be used to detect neurogenesis in live animal and human models [4].

A new noninvasive method is necessary to evaluate neurogenesis and to aid the management of neurological diseases. We propose that brain scintigraphy after radiolabeling of a specific antibody of an immature neuronal marker is a useful new modality for neurogenesis detection.

\section{Neurogenesis distribution}

During embryonic development, continuous divisions of NPCs that exist in the periventricular region and around the spinal central canal terminally form the CNS [5]. After the embryonic period, many of these precursors leave the precursor cell cycle and eventually differentiate into astrocytes and oligodendrocytes that are associated with the formation of the ependymal single-cell layers of the periventricular zones [5]. New neurons are produced in 
the cerebellum and the brain stem nuclei for a short time early in the postnatal period [2].

In adults, the hippocampal dentate gyrus, the subependymal zone (SEZ), and the olfactory bulb are the main sites of neurogenesis. NPCs are also found in the neocortex, spinal cord, tegmentum, substantia nigra, amygdala, and brain stem of adults. Although the role of these NPCs in these brain regions is not clear, it seems that they enter the neurogenesis pathway after appropriate stimulation [1].

\section{Neurogenesis stage}

Neurogenesis occurs in several phases, including proliferation, migration, differentiation, survival, and integration of the new population of the neurons into the neural network [1]. New neurons are generated from bipotent radial glia-like stem cells (type-1 cells), which traverse two intermediate stages of neural development involving type- 2 and type- 3 cells and are terminally transformed to early postmitotic and to mature neurons [6].

Type- 1 cells are the early precursor population, which has morphological and antigenic properties similar to radial glia cells. Type- 1 cells usually have an apical process and the capability to penetrate the molecular layer [7]. The radial glia-like stem cells (type-1) exist in a large number of different areas of the CNS. They have a low propensity for proliferation, although some neurological diseases (e.g., hypoxia or seizures) can provoke proliferative activity of these type- 1 cells [8].

Type- 2 cells are intermediate precursor cells, with high proliferative activity [8]. Type-2 cells demonstrate overlap in glial (nestin) and neuronal marker expression (doublecortin [DCX] and the polysialylated neural cell adhesion molecule [PSA-NCAM]) [7].

The type- 3 cell stage involves a shift from a "neuroblast," with slow proliferative activity, to a postmitotic immature neuron. Usually, type-3 cells exhibit only slight proliferative activity. In contrast to type-2 cells, type- 3 cells do not express glial markers, but they do express neuronal lineage markers (DCX, PSA-NCAM, neuroD, Prox1). The developmental transition of type-3 cells is dynamic, with processes of different lengths and components and various directions that constantly change from horizontal to vertical. The termination of the cell cycle mainly takes place at the type3 cell stage, with the temporary expression of the calcium-binding protein calretinin [7].

\section{Neurogenesis markers}

NeuroD

NeuroD is a basic helix-loop-helix transcription factor, which plays a basic role in the regulation of the cell cycle and neuronal differentiation [9]. NeuroD is predominantly associated with early aspects of neuronal differentiation [10].

The protein is expressed in a specific population of neuronal cells in the central and peripheral nervous systems. Its expression is transient and is limited to the terminal differentiation and generation of mature neurons [11]. The expression of neuroD1 is considered one of the earliest markers of granule cell development [8].The ventricular zone stem cells do not express neuroD because the protein does not have a role in the early stages of neurogenesis (e.g., proliferation and migration) [10]. Steiner et al. evaluated neurogenesis stage in adult mice hippocampal and showed expression of neuroD protein by anti-NeuroD1 on type- 2 cells in the early stages of neurogenesis [8].

During the early postnatal period, the adult neocortex has a low level of expression of neuroD mRNA, making it difficult to detect the protein by the in situ hybridization method [10]. However,
neuroD is continuously expressed in a specific subset of mature neurons in the adult brain, including pyramidal neurons in the CA1-CA3 region of the hippocampus, granule cells of the dentate gyrus, and granule cells of the cerebellum [10] (Fig. 1).

\section{Doublecortin}

DCX is a protein involved in the binding and stabilization of microtubules. It participates in different events related to the neuronal cytoskeleton, including the migration stage. Similar to neuroD, DCX is a neurogenesis marker [4]. Brown et al. investigated the temporal expression pattern of DCX by Immunofluorescence method in neurogenic regions of the adult rat brain [4]. The expression of DCX during the neuron proliferation period increases on days 4-7 after BrdU labeling and then rapidly decreases. The amount of DCX falls to a low level 1 month after labeling (about $2 \%$ ) and by 2 months, it is undetectable [4].

The expression of DCX coincides with the migration of neuronal stem cells throughout the development of the CNS. In the embryonic brain, DCX is expressed in a high concentration, and it can be identified in most embryonic intermediate zone and cortical plate cells. In the embryonic period, DCX is expressed mainly by neuronal precursors and during radial and tangential migration [12].

Although the expression of DCS is limited within the adult brain, some neurogenic regions, including the hippocampal dentate gyrus, the SEZ, and the olfactory bulb, preserve DCX expression . In addition to immature neurons, mature neurons in nonneurogenic areas, including the cortex, the striatum, and the corpus callosum, express DCX, although in very low amounts. Brief DCX expression outside the neurogenic area is attributed to probable neurogenesis processes in the region and to structural plasticity, such as sprouting [4,13] (Fig. 1).

\section{Polysialylated neural cell adhesion molecule}

The carbohydrate portion on the cell surface of PSA-NCAM participates in different stages of CNS development [14]. PSA-NCAM is mainly expressed during CNS development, but populations of mature interneurons in the hippocampus and in the prefrontal cortex express PSA during adulthood [15].

The marker is expressed by neuroblasts and young neurons. Compared with DCX, it appears to be expressed later and for a longer time [12]. Type-1 cells do not express PSA-NCAM [6]. Although PSA-NCAM is a maker of neurogenesis, mature neurons can also express this molecule in specific circumstances, such as synaptic plasticity [12] (Fig. 1).

\section{Calretin}

Calretin is a calcium-binding protein and neurogenesis marker. Brandt et al. evaluated expression of calretin in adult dentate gyrus by Immunohistochemistry method and showed which the protein is abundantly expressed between day three and the first week after cell division [16]. The expression of calretin can be detected 1 day after labeling of divided cells with BrdU, and it is not detectable before this time. Early after BrdU, the molecule associated with DCX express by immature neuron cells. However, it persists for a longer period than DCX, and its expression overlaps with that of NeuN, a mature neuronal marker. After 6 weeks, calretin is no longer expressed in new neurons [16] (Fig. 1).

\section{Neurogenesis and neurological disorders}

Several neuropsychiatric conditions, such as stroke, mechanical injury, Alzheimer's disease, Parkinson's disease, Huntington's 


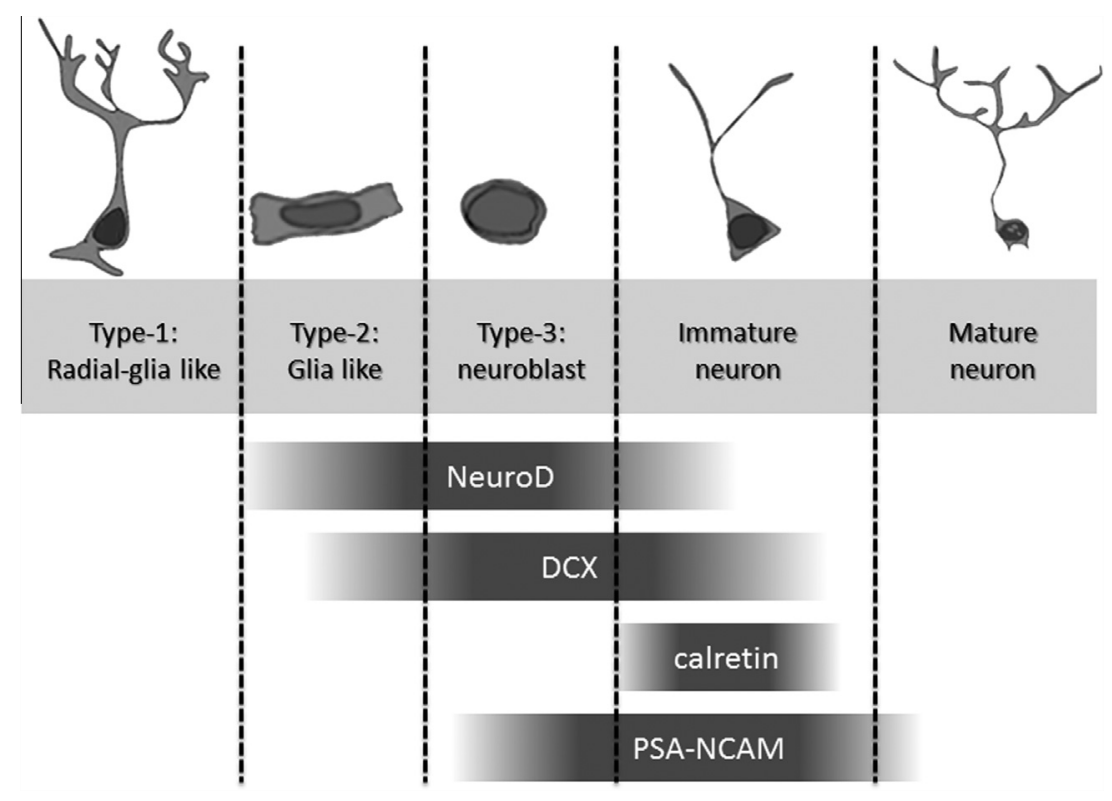

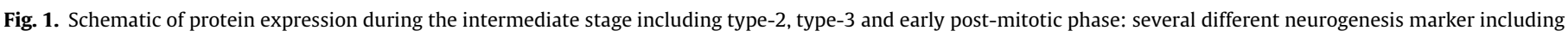

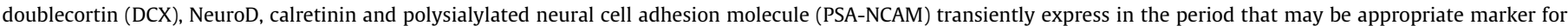
neurogenesis determination and monitoring.

disease, and amyotrophic lateral sclerosis, are associated with altered rates of neurogenesis in animal models [1].

Immature neuron markers, such as DCX, PSA-NCAM, and neuroD, are expressed in the hippocampus of patients with Alzheimer's disease, and the number of cells expressing these molecules also increases, consistent with enhanced neurogenesis in human Alzheimer's disease [17].

Brain ischemia increases the proliferation of new neurons in hippocampal and SVZ regions of adult animals [1]. Neurogenesis in the hippocampus and the olfactory bulb after cerebral mechanical damage also stimulates the production of new neurons in other sites affected by the injury [1].

\section{Hypothesis}

Brain PET or SPECT scintigraphy after immature neuronal marker radiolabeling may detect neurogenesis.

As discussed above, neurogenesis is limited in the adult brain and restricted to specific areas of the brain, although several environmental and pathological factors (e.g., stress, exercise, ischemia, Alzheimer's disease) modulate and increase neurogenesis in other areas affected by neuronal injury and loss. Currently, neurogenesis detection depends on invasive immunohistochemistry, which is not applicable to live animal and human models. We propose that immature neuronal markers, such as neuroD, DCX, or calretinin, targeted with a specific radiolabeled antibody, may show neurogenesis in a live environment. Radiolabeled antibodies have trivial radioactive materials attached to them [18-20]. $99 \mathrm{~m}$ Tc-IgG and 111In-CYT-103 are instances of radiolabeled antibodies [18-20]. Such radiolabel antibodies have low radiation burden [18-20].

A suitable marker for this purpose should have specific properties: It should be expressed alone in intermediate stages of neuron development. Including type- 2 and type- 3 cells [6] because the expression of neuron markers in this period is transient and limited to the period during which neurogenesis is induced. It seems the markers are not expressed in usual situation. Markers related to precursor cells are not suitable for neurogenesis detection because they are normally expressed in other areas of the brain, such as the SVZ and the hippocampus.
Recently, Rueger et al. used positron emission tomography (PET) and the radiotracer 3-deoxy-3-[18F]fluoro-L-thymidine ([18F]FLT) that enables imaging and measuring of proliferation to noninvasively detect endogenous neural stem cells in the normal and diseased adult rat brain in vivo. This method indeed visualized neural stem cell niches in the living rat brain, identified as increased [18F]FLT-binding in the SVZ and the hippocampus. After phosphorylation by TK1, [18F]FLT is trapped within the cell and can therefore be visualized by PET [21].

\section{Clinical implications}

1. Neurogenesis, together with neuronal loss, is present in various neurodegenerative diseases, with the rate of neurogenesis lower than the rate of neuronal loss.

2. Adult neurogenesis can be stimulated by many molecular factors, including basic fibroblast growth factor, epidermal growth factor, insulin-like growth factor 1, and brain-derived neurotrophic factor [22].

Clinical evaluation may be a reliable and noninvasive way of assessing the effect of different neurogenesis-inducing factors on neurological disorders. However, the proposed noninvasive imaging method, if proven, may enable direct measuring of neurogenesis and replace traditional invasive methods, which have limited capability in live models. The detection of neurogenesis by scintigraphy imaging can lead to a revolution in treatment in the neurological field [23].

\section{Conflicts of interest}

The author(s) declare that they have no conflict of interests.

\section{References}

[1] Yoneyama M, Shiba T, Hasebe S, Ogita K. Adult neurogenesis is regulated by endogenous factors produced during neurodegeneration. J Pharmacol Sci 2011;115(4):425-32. 
[2] Kaslin J, Ganz J, Brand M. Proliferation, neurogenesis and regeneration in the non-mammalian vertebrate brain. Philos Trans $\mathrm{R}$ Soc Lond B Biol Sci 2008;363(1489):101-22.

[3] Tanaka R, Yamashiro K, Mochizuki H, Cho N, Onodera M, Mizuno Y, et al. Neurogenesis after transient global ischemia in the adult hippocampus visualized by improved retroviral vector. Stroke 2004;35(6):1454-9.

[4] Brown JP, Couillard-Després S, Cooper-Kuhn CM, Winkler J, Aigner L, Kuhn HG. Transient expression of doublecortin during adult neurogenesis. J Comp Neurol 2003;467(1):1-10.

[5] Kazanis I. The subependymal zone neurogenic niche: a beating heart in the centre of the brain: how plastic is adult neurogenesis? Opportunities for therapy and questions to be addressed. Brain 2009;132(Pt 11):2909-21.

[6] Kempermann G, Jessberger S, Steiner B, Kronenberg G. Milestones of neuronal development in the adult hippocampus. Trends Neurosci 2004 Aug;27(8):447-52.

[7] Miller FD, Gauthier-Fisher A. Home at last: neural stem cell niches defined. Cell Stem Cell 2009;4(6):507-10.

[8] Steiner B, Klempin F, Wang L, Kott M, Kettenmann H, Kempermann G. Type-2 cells as link between glial and neuronal lineage in adult hippocampal neurogenesis. Glia 2006;54(8):805-14.

[9] Ochocinska MJ, Hitchcock PF. NeuroD regulates proliferation of photoreceptor progenitors in the retina of the zebrafish. Mech Dev 2009;126(3-4):128-41.

[10] Schwab MH, Druffel-Augustin S, Gass P, Jung M, Klugmann M, Bartholomae A, et al. Neuronal basic helix-loop-helix proteins (NEX, neuroD, NDRF): spatiotemporal expression and targeted disruption of the NEX gene in transgenic mice. J Neurosci 1998:18(4):1408-18.

[11] Lee JE, Hollenberg SM, Snider L, Turner DL, Lipnick N, Weintraub H. Conversion of Xenopus ectoderm into neurons by NeuroD, a basic helix-loop-helix protein. Science 1995;268(5212):836-44.

[12] Francis F, Koulakoff A, Boucher D, Chafey P, Schaar B, Vinet MC, et al. Doublecortin is a developmentally regulated, microtubule-associated protein expressed in migrating and differentiating neurons. Neuron 1999;23:247-56.

[13] Nacher J, Crespo C, McEwen BS. Doublecortin expression in the adult rat telencephalon. Eur J Neurosci 2001;14:629-44.
[14] Seki T. Expression patterns of immature neuronal markers PSA-NCAM, CRMP4 and NeuroD in the hippocampus of young adult and aged rodents. J Neurosci Res 2002 Nov 1;70(3):327-34.

[15] Gómez-Climent MÁ, Guirado R, Castillo-Gómez E, Varea E, Gutierrez-Mecinas M, Gilabert-Juan J, et al. The polysialylated form of the neural cell adhesion molecule (PSA-NCAM) is expressed in a subpopulation of mature cortica interneurons characterized by reduced structural features and connectivity. Cereb Cortex 2011 May;21(5):1028-41.

[16] Brandt MD, Jessberger S, Steiner B, Kronenberg G, Reuter K, Bick-Sander A et al. Transient calretinin expression defines early postmitotic step of neurona differentiation in adult hippocampal neurogenesis of mice. Mol Cell Neurosc 2003 Nov;24(3):603-13.

[17] Jin K, Peel AL, Mao XO, Xie L, Cottrell BA, Henshall DC, et al. Increased hippocampal neurogenesis in Alzheimer's disease. Proc Natl Acad Sci USA 2004;101(1):343-7.

[18] Asli IN, Javadi H, Seddigh H, Mogharrabi M, Hooman A, Ansari M, et al. The diagnostic value of $(99 \mathrm{~m}) \mathrm{Tc}$-IgG scintigraphy in the diabetic foot and comparison with (99 m)Tc-MDP scintigraphy. J Nucl Med Technol 2011 Sep;39(3):226-30.

[19] Rubin RH, Fischman AJ. The use of radiolabeled nonspecific immunoglobulin in the detection of focal inflammation. Semin Nucl Med 1994 Apr;24(2):169-79.

[20] Doerr RJ, Abdel-Nabi H, Krag D, Mitchell E. Radiolabeled antibody imaging in the management of colorectal cancer. Results of a multicenter clinical study. Ann Surg 1991 Aug;214(2):118-24.

[21] Rueger MA, Backes H, Walberer M, Neumaier B, Ullrich R, Simard ML, et al. Noninvasive imaging of endogenous neural stem cell mobilization in vivo using positron emission tomography. J Neurosci 2010 May 5;30(18):6454-60.

[22] Chopp M, Zhang ZG, Jiang Q. Neurogenesis, angiogenesis, and MRI indices of functional recovery from stroke. Stroke 2007 Feb;38(2 Suppl.):827-31.

[23] Nemati R, Mehdizadeh S, Nabipour I, Amini A, Assadi M. Radiolabeled antiaquaporin-4 protein antibody imaging: a valuable method for determining neuromyelitis optica. Nucl Med Commun 2013 May;34(5):413-6. 